\title{
Effect of Androgen Deprivation Therapy on Indices of Glycaemia and Insulin Resistance in Native African Prostate Cancer Patients
}

\author{
lya Eze Bassey*, Chinyere Adanna Opara Usoro and Kaiso Sunday Umo Esiere
}

Department of Medical Laboratory Sciences, Faculty of Allied Medical Sciences, College of Medical Sciences, University of Calabar, Calabar, Nigeria

${ }^{*}$ Corresponding author: Iya Eze Bassey, Department of Medical Laboratory Sciences, Faculty of Allied Medical Sciences, College of Medical Sciences, University of Calabar, Calabar, Nigeria, Tel: +2348035472034; E-mail: iyantui@yahoo.com

Received: August 26, 2017; Accepted: September 04, 2017; Published: September 12, 2017

Citation: Bassey IE, Usoro CAO, Esiere KSU (2017) Effect of Androgen Deprivation Therapy on Indices of Glycaemia and Insulin Resistance in Native African Prostate Cancer Patients. J Neoplasm Vol.2 No.2:10.

\section{Abstract}

Background: Hyperglycemia and insulin resistance have been associated with androgen deprivation therapy (ADT) in Caucasian populations. It is therefore relevant to determine this also holds true in a native African population.

Methods and Findings: The design of the study was cross sectional, 153 prostate cancer patients and 80 controls were recruited. Prostate specific antigen (PSA), insulin and glucose were determined using ELISA and colorimetric methods. Homeostasis assessment model for insulin resistance (HOMA IR) and HOMA2 IR were calculated. The prostate cancer patients were sub-grouped into treatment-naive prostate cancer cases and those on androgen deprivation therapy. Statistical analysis was carried out using Analysis of variance and least significant difference, $p<0.05$ was considered statistically significant. There was no significant variation $(p>0.05)$ in the mean insulin, glucose, HOMA IR and HOMA2 IR among the treatment-naive prostate cancer patients and prostate cancer patients on treatment and controls. In addition, there were no significant variations $(p>0.05)$ in these parameters among patients treated with different types and durations of ADT. There was no significant correlation ( $p>0.05$ ) between PSA and insulin, glucose, HOMA IR and groups HOMA2 IR in any of the groups.

Conclusion: Androgen deprivation therapy does not result in elevated blood glucose, insulin and insulin resistance in native African men.

Keywords: Prostate cancer; African men; Insulin; Glucose; Insulin resistance

\section{Introduction}

Prostate Cancer (CaP) continues to be the most significant cancer that has disproportionately overburdened men of African ancestry [1]. It has been reported that CaP is the most common cancer in Nigerian men, and accounts for $6.1 \%$ to $19.5 \%$ of all cancers in the country and its incidence is still on the increase [2]. The average prevalence in Nigeria has been reported to be about $11 \%$ [3].

For now, Androgen Deprivation Therapy (ADT) is the most commonly used therapy for prostate cancer and its use has been associated with increased survival rates in many of the patients [4]. Due to its association with numerous side effects, it has been suggested in a recent studies that treatment plans for patients with prostate cancer being treated with androgen deprivation therapy should include screening for cardiovascular risk factors $[5,6]$.

In several studies on predominantly Caucasian populations and a few on racially mixed populations, androgen deprivation therapy (ADT) has been shown to increase insulin resistance $[7,8]$ alter glycemic control causing hyperglycemia $[9,10]$ and may contribute to development of Type 2 diabetes in the longterm [10-14].

Despite evidence suggesting unfavorable alteration of indices of glycaemia in prostate cancer patients undergoing $A D T$, there are very limited if any research on this subject in native African prostate cancer patients and therefore in Nigerian men; and thus the need for this study. This study examined the effect of ADT on indices of glycemia and insulin resistance in Nigerian prostate cancer patients undergoing ADT.

\section{Materials and Methods}

\section{Study design and subject selection}

The design of the study was cross sectional consisting of two hundred and thirty three (233) participants who were consecutively recruited. They consisted of 153 patients of Nigerian origin with confirmed prostate cancer attending the Surgical Out Patient Clinic of the University of Calabar Teaching Hospital and 80 age-matched apparently healthy male subjects without prostate cancer or BPH as controls from the Calabar metropolis. The prostate cancer patients were grouped into the ADT-treated group and the treatment-naïve group (these were patients with prostate cancer who had just been 
diagnosed and who had not started ADT or any other treatment). Surgical castration and Medical castration (GnRH, biclutamide, and flutamide) were the ADT treatment modalities employed. The treated group was also sub grouped according to their duration of treatment into those who had undergone ADT for up to one year and those who had undergone $A D T$ for more than one year. Fasting plasma glucose, serum insulin and PSA were estimated samples obtained from them. Homeostasis assessment model for insulin resistance (HOMA1 IR and HOMA2 IR) was calculated.

This study was conducted in accordance with the World Medical Association's Declaration of Helsinki [15]. The purpose and nature of the research was explained each participant and informed consent obtained from them. A standard questionnaire was administered to them to obtain information from the subjects about their age, family history, dietary and physical lifestyle.

\section{Inclusion criteria}

All the subjects were male and aged 45 yrs and above. The controls did not have prostate cancer or $\mathrm{BPH}$.

\section{Exclusion criteria}

Terminally ill prostate cancer patients and participants who refused to participate were excluded.

\section{Sample size calculation}

The number of samples in this research was determined using the Leslie Kish formulae [16],

$$
N=\frac{Z a^{2} p q}{d^{2}}
$$

where,

\section{$\mathrm{N}=$ desired sample size}

$Z \alpha=$ the $\alpha$ level of the coefficient interval at 95\% (1.96)

$\mathrm{p}=$ proportion of occurrence

$q=(1-p)$ proportion of non-occurrence

$\mathrm{d}=$ precision

Substituting the expected occurrence of $p=11 \%$ from Akinremi, et al. [3] i.e., 0.11 we have

$$
N=\frac{1.962 \times 0.11(1-0.11)}{(0.05)^{2}}=150.4
$$

\section{Sample collection}

Blood samples were aseptically collected from each subject between 8 a.m. and 10 a.m. after an overnight fast and after 3 days abstinence from alcohol and sexual activities. Five milliliters of blood was aseptically collected; $3 \mathrm{ml}$ of blood was put in plain bottles, left to clot and the serum extracted and frozen till used while $2 \mathrm{ml}$ of blood was put in fluoride oxalate bottles and centrifuged to obtain plasma for glucose estimation.

\section{Estimation of plasma glucose serum PSA and insulin}

Plasma glucose was estimated by enzymatic method of Barham and Trinder [17]. Insulin was determined with an ELISA kit obtained from DRG $\mathrm{GmBH}$, Germany. Prostate specific antigen ELISA kit was obtained from Syntron Bioresearch, USA.

\section{Calculation of homeostasis assessment model for insulin resistance (HOMA IR)}

HOMA IR was calculated using Homeostasis assessment model for insulin resistance (HOMA1-IR) was calculated as: Fasting plasma glucose $(\mathrm{mmol} / \mathrm{l}) \times$ Fasting plasma insulin $(\mu \mathrm{U} / \mathrm{ml}) / 22.5$ [18] and HOMA2 IR was calculated using the HOMA2 Calculator v2.2.3, a software by the Diabetes Trials Unit, University of Oxford, UK.

\section{Statistical analysis}

Statistical analysis was performed using the PAWstatistic 18, a statistical package from SPSS Inc, California, USA. The results were expressed as Mean \pm SD. The data was analyzed by Student's t-test and analysis of variance (ANOVA). Post-hoc analysis was done using Least Significant Difference (LSD). The level of significance was set at $95 \%$ confidence interval, where $p$-value less than $0.05(p<0.05)$ was considered as statistically significant.

\section{Results}

When mean prostate specific antigen, insulin, glucose, HOMA1-IR, HOMA2 IR were compared in treatment-naive prostate cancer patients and prostate cancer patients on ADT and controls, there was only a significant variation in the PSA levels among the groups. There was no significant variation in the other parameters. As expected the treatment-naive group had the highest PSA values (Table 1).

Table 1 Mean prostate specific antigen, insulin, glucose, HOMA1-IR, HOMA2 IR in treatment-naive prostate cancer patients and prostate cancer patients on androgen deprivation

\begin{tabular}{|c|c|c|c|c|}
\hline \multirow[t]{2}{*}{ Parameter } & $\begin{array}{l}\text { ADT } \\
\text { treated } \\
\text { CaP } \\
\text { patients }\end{array}$ & $\begin{array}{l}\text { Treatment- } \\
\text { naive CaP } \\
\text { patients }\end{array}$ & Controls & \multirow[t]{2}{*}{ p-value } \\
\hline & $n=111$ & $n=42$ & $n=80$ & \\
\hline PSA (ng/ml) & $\begin{array}{l}23.52 \pm \\
30.54 \#\end{array}$ & $\begin{array}{ll}52.84 & \pm \\
31.49^{\star} \# & \end{array}$ & $1.15 \pm 1.01$ & 0.0001 \\
\hline Insulin $(\mu \mid U / L)$ & $\begin{array}{l}14.87 \quad \pm \\
14.31\end{array}$ & $11.80 \pm 11.62$ & $\begin{array}{l}12.46 \\
12.27\end{array}$ & 0.303 \\
\hline $\begin{array}{l}\text { Glucose } \\
(\mathrm{mmol} / \mathrm{L})\end{array}$ & $\begin{array}{l}4.56 \quad \pm \\
1.51\end{array}$ & $5.08 \pm 2.40$ & $4.60 \pm 2.34$ & 0.336 \\
\hline
\end{tabular}
therapy and controls. 


\begin{tabular}{|c|lr|c|c|c|}
\hline HOMA1 IR & $\begin{array}{l}3.45 \\
4.35\end{array}$ & \pm & $2.84 \pm 3.61$ & $2.60 \pm 2.79$ & 0.289 \\
\hline HOMA2 IR & $\begin{array}{l}1.84 \\
1.78\end{array}$ & \pm & $1.49 \pm 1.50$ & $1.51 \pm 1.43$ & 0.285 \\
\hline
\end{tabular}

Results expressed as MEAN $\pm \mathrm{SD}$; HOMA1 IR: Homeostasis Assessment Model 1 for Insulin Resistance; HOMA2 IR: Homeostasis Assessment Model 2 for Insulin Resistance; *: Significantly higher than that of ADT treated CaP patients; \#: Significantly higher than that of controls.

Table 2 shows the effect of surgical and medical castration on prostate specific antigen, glucose, insulin, HOMA1 IR and HOMA2 IR levels in prostate cancer patients. Except for PSA, there was no significant variation in the other parameters among the groups. Both treatment groups had lower PSA levels compared to the treatment-naive group.

Table 2 Effect of surgical and medical castration on prostate specific antigen, glucose, insulin, HOMA IR and HOMA2 IR levels in prostate cancer patients.

\begin{tabular}{|c|c|c|c|c|}
\hline Parameter & $\begin{array}{l}\text { Medical } \\
\text { castration }\end{array}$ & $\begin{array}{l}\text { Surgical } \\
\text { castration }\end{array}$ & $\begin{array}{l}\text { Treatment- } \\
\text { naive CaP } \\
\text { patients }\end{array}$ & $\begin{array}{l}p- \\
\text { value }\end{array}$ \\
\hline & $n=71$ & $n=40$ & $n=42$ & \\
\hline PSA (ng/ml) & $22.6 \pm 31.56$ & $25.2 \pm 28.94$ & $\begin{array}{l}52.84 \\
31.49^{\star} \#\end{array}$ & 0.0001 \\
\hline Insulin (IU/L) & $14.1 \pm 13.76$ & $15.1 \pm 14.76$ & $11.80 \pm 11.62$ & 0.455 \\
\hline $\begin{array}{l}\text { Glucose } \\
\text { (mmol/L) }\end{array}$ & $4.67 \pm 1.70$ & $4.37 \pm 1.10$ & $5.08 \pm 2.40$ & 0.2 \\
\hline HOMA1 IR & $3.65 \pm 4.78$ & $3.08 \pm 3.67$ & $2.88 \pm 3.59$ & 0.607 \\
\hline HOMA2 IR & $1.88 \pm 1.84$ & $1.73 \pm 1.70$ & $1.49 \pm 1.51$ & 0.508 \\
\hline
\end{tabular}

Results expressed as Mean \pm SD; HOMA1 IR: Homeostasis Assessment Model 1 for Insulin Resistance; HOMA2 IR: Homeostasis Assessment Model 2 for Insulin Resistance; *: Significantly higher than that of Medical castration; \#: Significantly higher than that of Surgical castration.

The effect of duration of treatment on prostate specific antigen, glucose, insulin, HOMA1 IR and HOMA2 IR in treatment-naive prostate cancer patients and prostate cancer patients undergoing ADT for up to a year and more than a year was also examined. There was only a significant variation in the PSA levels among the groups. There was no significant variation in the other parameters. There was no significant correlation between PSA and the other parameters in any of the groups.

\section{Discussion}

Hyperglycemia and insulin resistance have been associated with androgen deprivation therapy (ADT) in Caucasian populations. This results in an increase in new-onset diabetes mellitus thereby creating a double burden of disease in this category of patients. A consequence of this may be decreased survival rate or/and quality of life.

Our study showed no significant variations $(p>0.05)$ in the plasma levels of fasting insulin, glucose HOMA1 IR, HOMA2 IR among treatment-naive prostate cancer patients and prostate cancer patients on ADT and controls. This was different from reports from other studies done in Caucasians [8,12,19-22]and racially mixed studies [11] in the USA. They reported that men who undergo ADT have a greater risk of developing hyperglycemia and insulin resistance. This may be due to the differences between populations. It is good to note that our study was on native African men while these studies were carried out in mostly Caucasian populations. Though most studies have shown that African Americans have higher risk of having type 2 diabetes compared to Caucasians, this may not be the case for native Africans living more of a traditional lifestyle (i.e., eating traditional diets and an active lifestyle) than a westernized one [23]. This is because though genetic background of Africans makes them more differentially susceptible to diabetes than Caucasians [24], extraneous factors such as diet, lifestyle and environment may modify the expression of this susceptibility. To support this, it has also been shown that the prevalence of diabetes in native Africans is much lower than that for men of African ancestry living in the USA or the UK [25]. However, in a study on native Hispanic men, no significant difference also was observed in the mean glucose levels of prostate cancer patients on ADT and prostate cancer patients not on ADT [26].

It is interesting that both the duration and type of ADT did not show any effect on the glucose, insulin or any of the measures of insulin resistance in our study. In contrast, in a prospective study on non-diabetic men with prostate cancer on ADT, it was observed that after 3 months of ADT, fasting plasma insulin levels increased by $25.9 \%$ and mean insulin sensitivity decreased by $12.8 \%$ from baseline $[12,19]$ reported that short-term ADT (within 3 to 6 months) causes the development of hyperinsulinemia while long-term $(\leq 12$ months) ADT results in higher prevalence of diabetes when compared with controls. We did not observe this in our study.

A limitation of this study is the number of participants involved. A larger cohort which is prospective in nature involving both native born and immigrant Africans and taking into account lifestyle factors, diet, and physical activity may be able to explain the disparities observed.

\section{Conclusion}

Androgen deprivation therapy does not result in elevated blood glucose, insulin and insulin resistance in native African men.

\section{Acknowledgements}

We wish to thank all the consultant urologists of the University of Calabar for allowing us access to their patients especially to Dr. Enakirerhi E. Glen for his useful insights in this study.

\section{Funding}

This study was self-funded. 


\section{Conflicting Interests}

All authors declare no conflict of interest.

\section{References}

1. American Cancer Society (2016) Cancer Facts $a^{\text {nd }}$ Figures. American Cancer Society Atlanta, GA.

2. http://nigeriahealthwatch.com/wp-content/uploads/bsk-pdf-manager/ 597_598_2009_EPIDEMIOLOGY_OF_COMMON_CANCERS_IN_NIGERIA,_FATIMA_AB DULKAREEM.PDF

3. Akinremi TO, Ogo CN, Olutunde AO (2011) Review of prostate cancer research in Nigeria. Infect Agents Cancer 6: S8.

4. Gunnarsson O, Basaria S, Gignac GA (2015) Cardiometabolic and skeletal risk factors in black men with prostate cancer starting androgen deprivation therapy. Cancers 7: 679-687.

5. Keating NL, O'Malley JA, FreedlandSJ, Smith MR (2010) Diabetes and cardiovascular disease during androgen deprivation therapy: Observational study of veterans with prostate cancer. J Natl Cancer Inst. 102: 39-46.

6. Essien OE, Bassey IE, Gali RM, Udoh AE, Akpan UO, et al. (2017) Cardiovascular disease risk factors: How relevant in African men with prostate cancer receiving androgen-deprivation therapy? J Glob Oncol 3: 7-14.

7. Haider A, Yassin A, Saad F, Shabsigh R (2007) Effects of androgen deprivation on glycaemic control and on cardiovascular biochemical risk factors in men with advanced prostate cancer with diabetes. Aging Male 10: 189-196.

8. Basaria S, Muller DC, Carducci MA, Egan J, Dobs AS (2006) Hyperglycemia and insulin resistance in men with prostate carcinoma who receive androgen deprivation therapy. Cancer 106: $581-588$

9. Nishiyama T, Ishizaki F, Anraku T, Shimura H, Takahashi K (2005) The influence of androgen deprivation therapy on metabolism in patients with prostate cancer. J Clin Endocrinol Metab 90: 657-660.

10. Yannucci J, Manola J, Garnick MB, Bhat G, Bubley GJ (2006) The effect of androgen deprivation therapy on fasting serum lipid and glucose parameters. J Urol 176: 520-525.

11. Derweesh IH, Diblasio CJ, Kincade MC, Malcolm JB, Lamar KD, et al. (2007) Risk of new-onset diabetes mellitus and worsening glycaemic variables for established diabetes in men undergoing androgen-deprivation therapy for prostate cancer. BJU Int 100: 1060-1065.

12. Shahani S, Braga-Basaria M, Basaria S (2008) Androgen deprivation therapy in prostate cancer and metabolic risk for atherosclerosis. J Clin Endocrinol Metab. 93: 2042-2049.
13. Traish AM, Saad F, Guay A (2009) The dark side of testosterone deficiency, II. Type 2 diabetes and insulin resistance. J Androl 30: 23-32.

14. Wang H, Sun X, Zhao L, Chen X, Zhao J (2016) Androgen deprivation therapy is associated with diabetes: Evidence from meta-analysis. J Diabetes Investig 7: 629-636.

15. World Medical Association (2013) Declaration of Helsinki: Ethical principles for medical research involving human subject. JAMA 310: 2191-2194.

16. Kish L (1965) Survey Sampling. Wiley New York, USA.

17. Barham D, Trinder P (1972) An improved colour reagent for the determination of blood glucose by the oxidase system. Analyst. 97: $142-{ }^{145}$.

18. Matthews DR, Hosker JP, Rudenski AS, Naylor BA, Treacher DF, et al. (1985) Homeostasis model assessment: insulin resistance and beta-cell function from fasting plasma glucose and insulin concentrations in man. Diabetologia 28: 412-419.

19. Smith $M R$, Lee $H$, Nathan DM (2006) Insulin sensitivity during combined androgen blockade for prostate cancer. J Clin Endocrinol Metab 91: 1305 -1308.

20. Hammarsten J, Högstedt B (2004) Clinical, hemodynamic, anthropometric, metabolic and insulin profile of men with high stage and high grade clinical prostate cancer. Blood Press 13: 47-55.

21. Hammarsten J, Högstedt B (2005) Hyperinsulinemia: A prospective risk factor for lethal clinical prostate cancer. Eur J Cancer 41: 2887-2895.

22. Hammarsten J, Damber JE, Peeker R, Mellström D, Högstedt BA (2010) Higher prediagnostic insulin level is a prospective risk factor for incident prostate cancer. Cancer Epidemiol 34: 574-579.

23. Admiraal W, Holleman F (2014) Ethnic aspects of epidemiology. Diapedia 11040851199 rev. no. 32.

24. Kodama K, Tojjar D, Yamada S, Toda K, Patel CJ, et al. (2013) Ethnic differences in the relationship between insulin sensitivity and insulin response: A systematic review and metaanalysis. Diabetes Care 36: 1789-1796.

25. International Diabetes Federation (2006) IDF diabetes atlas. (3rd edn). Belgium

26. Cleffi S, Neto AS, Reis LO, Maia P, Fonseca F, et al. (2011) Androgen deprivation therapy and morbid obesity: Do they share cardiovascular risk through metabolic syndrome? Actas Urol Esp 35: 259-265. 